\title{
Transferencia de conocimiento como factor crítico para la gestión de la ciencia, la tecnología y la innovación en Maloka Bogotá - Colombia
}

\author{
Carlos, E. Blanco-Valbuena
}

Wilmer Pineda ${ }^{2}$

Recibido: 07-02-2019

Aceptado: 16-02-2019

\section{Resumen}

Objetivo. Evaluar las variables de cultura organizacional, las características del conocimiento tácito-explícito y las estrategias de transferencia interna de conocimiento, por incidir en la gestión de Maloka en relación con su fin: enriquecer la cultura ciudadana estableciendo lazos entre ciencia-tecnología- innovación y sociedad. Metodología. Buscando reducir dimensiones, se aplicó el análisis factorial como técnica estadística que define grupos de variables altamente correlacionados entre sí. Hemos utilizado el test de KMO (Kaiser-Meyer-Olkin) para saber si la técnica de reducción de dimensiones es viable. El estudio se ha hecho con una muestra de 72 directivos de niveles alto y medio de Maloka, corporación de gran visibilidad nacional e internacional. Resultados. Las variables de cultura organizacional evidencian la existencia de gran confianza basada en valores morales y éticos; en las creencias colectivas;

1. Ing. Dr. Economía y Dirección de Empresas, Universidad de Deusto, País Vasco. Profesor-Investigador, Investigador principal, Facultad de Ciencias y Tecnologías, Universidad Santo Tomás de Bogotá.

Bogotá, Colombia.

Correo electrónico: carlosblancov@usantotomas.edu.co - scarlose.co2010@gmail.com ORCID: http://orcid.org/0000-0001-9490-7604

Google Académico https://scholar.google.es/citations? user=SIdWYgkAAAAJ\&hl=es\&authuser=1 CVLac: http://scienti.colciencias.gov.co:8081/cvlac/visualizador/generarCurriculoCv.do?cod_ $\mathrm{rh}=0001110977$

2. Docente lider de investigación. PhD(c). Facultad de estadística. Universidad Santo Tómas. Bogotá, Colombia.

ORCID: http://orcid.org/0000-0001-7774-951X 
en las identidades de pensamiento surgidas de acontecimientos que conducen a la transferencia interna de conocimiento, y en los diálogos dirigidos a transferir los aprendizajes. La transferencia interna de conocimiento tácito se lleva a cabo a través de la observación directa a los expertos. El aprendizaje individual para la transferencia interna de conocimiento, se realiza a partir de estudiar lo escrito en las "historias que se aprenden". Las estrategias para transferir el conocimiento tácito se basan fundamentalmente en compartir experiencias por mentoría, entrenamiento, redes y uso de un lenguaje común, verbal o corporal.

Palabras clave: transferencia de conocimiento, conocimiento tácito y explícito, cultura organizacional, gestión de conocimiento.

\title{
Knowledge transfer as a critical factor for the management of science, technology and innovation in Maloka Bogota Colombia
}

\begin{abstract}
Objective. Evaluate the variables of organizational culture, the characteristics of tacit-explicit knowledge and internal knowledge transfer strategies, for influencing the management of Maloka in relation to its purpose: enrich the culture of citizens by establishing links between science-technology-innovation and society. Methodology. In order to reduce dimensions, factor analysis was applied as a statistical technique that defines groups of highly correlated variables. We have used the KMO test (Kaiser-MeyerOlkin) to know if the technique of reduction of dimensions is viable. The study has been done with a sample of 72 managers of high and medium levels of Maloka, a corporation of great national and international visibility. Results. The organizational culture variables show the existence of great confidence based on moral and ethical
\end{abstract}


values; in collective beliefs; in the identities of thought arising from events that lead to the internal transfer of knowledge, and in dialogues aimed at transferring learning. The internal transfer of tacit knowledge is carried out through direct observation to the experts. Individual learning for the internal transfer of knowledge is done from studying what is written in the "stories that are learned". The strategies for transferring tacit knowledge are fundamentally based on sharing experiences through mentoring, training, networking and using a common language, verbal or corporal.

Keywords: knowledge transfer, tacit and explicit knowledge, organizational culture, knowledge management.

\section{Introducción}

Grant (1996) afirma que el conocimiento que posee una empresa es uno de los activos más cruciales e importantes para la competitividad. Spender (1996) argumenta que el conocimiento y la habilidad para generarlo constituyen el centro de la teoría de la empresa. Mucho de este conocimiento reside en su capital humano, por tanto, la selección, el desarrollo y la utilización de ese capital puede y debe ser gestionado para la creación de valor en la organización.

Con respecto a lo anterior, la particularidad de una firma consiste en desarrollar las habilidades destinadas a crear, transferir, ensamblar, integrar y explotar el conocimiento. Se trata de un proceso que hoy se reconoce y denomina "gestión del conocimiento" (Inkpen, 2000), que en realidad es una extensión de la teoría de la firma basada en los recursos. Tenido esto en cuenta, la gestión del conocimiento es mucho más que el simple movimiento de los datos o de la transferencia de la información. Consiste en integrar, transferir, tener acceso y acumular el conocimiento, y, en última instancia, desarrollar y retener la experiencia a través de la organización (Pelton, 1999, citado por Bender y Fish, 2000). Desde esta 
perspectiva, nos centramos en la transferencia de conocimiento considerada como una capacidad organizacional (Grant, 1996a; Kogut y Zander, 1992; Nonaka, 1994; Spender, 1996; Teece et al., 1997). En sus investigaciones, Hitt et al. (2001b) encontraron que la transferencia del conocimiento incorporada en el capital humano-capacidades de los empleados- contribuye a obtener un alto rendimiento. La transferencia de conocimiento es vista como una parte importante de su gestión (Davenport y Prusak, 1998; Nissen et al., 2000; Beijerse, 2000, Blanco-Valbuena y Peña, 2005; BlancoValbuena y Bernal, 2018).

La transferencia de conocimiento y su creación, unido a la experiencia en el marco de una "organización de aprendizaje", constituyen hoy en día el núcleo de aquellos factores considerados críticos para el éxito de la empresa. Según Marshall (1996), y Bender y Fish (2000), todo ello implica tener activada la transferencia de conocimiento y el logro de experiencias en todas las áreas y departamentos de la estructura de la organización, incluso de aquellas ubicadas fuera de las fronteras del propio país. En este sentido Garvin (1993), sugiere que, para aprender más, el conocimiento debe ser desarrollado, conservado y extendido con eficacia en toda la organización.

El proceso de transferencia interna de conocimiento no es, en modo alguno, una cuestión elemental. Como explica Seaton (2002), y Liyanage, Elhag, Ballal y Li (2009), requiere un tipo adicional de conocimiento: aquel acerca de cómo es eficazmente transmitido. Si no es así, el propósito de la transferencia se perderá si el conocimiento se transfiere desde la fuente al receptor sin contextualizar la forma en que debe ser utilizado por este último. Es el procedimiento completo el que puede ser identificado como transformación del conocimiento. La transformación denota capacidad de una organización para desarrollar y refinar las rutinas que facilitan la combinación de conocimiento existente con los conocimientos recién adquiridos y asimilados (Zahra y George, 2002).

Por lo ya expuesto, este estudio analiza los diferentes factores e indicadores que están relacionados con la transferencia interna 
de conocimiento como factor crítico para el éxito de Maloka. Esta Corporación, ubicada en Bogotá-Colombia, construye iniciativas de apropiación social de la Ciencia, la Tecnología y la Innovación, estrategias de fortalecimiento a la educación formal, experiencias itinerantes, procesos de educación democratizada y para toda la vida, cultura ciudadana, métodos de experimentación e inmersión para las familias, encuentros de formación y participación ciudadana.

Este artículo se compone de los siguientes apartados: En primer lugar, se presenta la revisión de la literatura a partir de los aportes de autores que han realizado trabajos, en transferencia de conocimiento, en contextos diferentes al caso de la Corporación Maloka, que es la que nosotros hemos utilizado; en segundo lugar, abordamos la metodología; en tercer lugar, centramos nuestra atención en la discusión de los resultados y, finalmente, en un cuarto, presentamos las conclusiones, las limitaciones y las recomendaciones con destino a futuras investigaciones.

En el presente trabajo, la revisión de la literatura se centra en recoger y presentar los cuatro elementos que constituyen el núcleo central de la investigación: el que se refiere al concepto de transferencia de conocimiento, el que se centra en la cultura organizacional más la transferencia de conocimiento, el que proyecta su interés sobre la naturaleza y las características del conocimiento tácito y explícito requeridas para posibilitar la transferencia, y, por último, el que aborda las estrategias para llevarla a cabo.

\section{Concepto de transferencia de conocimiento}

En opinión de Davenport y Prusak (1998) la transferencia implica tanto la transmisión de información a un receptor como la absorción y transformación por éste, sea cual sea su composición, individual o colectiva. McFadyen et al. (2009) consideran que la transferencia de conocimiento implica el movimiento de los hechos, las relaciones y las percepciones de una persona u organiza- 
ción a otra. Para Cristensen (2003) la transferencia no se limita a la explotación de los recursos accesibles, como es el conocimiento, sino que incide en el cómo adquirirlo y en el absorberlo adecuadamente para hacer las cosas más eficientes y eficaces. El fin es Ilevar a cabo acciones que permitan lograr un proceso más rápido y/o más seguro. De otra parte, la trasferencia, en el marco intelectual, es el flujo de conocimiento que se produce a través de los límites creados por dominios especializados, comunicando a otros lo que saben, o consultando a esos otros para adquirir lo que es de su dominio, logrando así, unos y otros, mejorar constantemente su propio saber, al tiempo que, de la manera más eficaz posible, es puesto a disposición de terceros para que puedan utilizarlo (Carlile y Rebentisch, 2003; Van den Hooff y De Ridder, 2004).

En opinión de Kumar y Ganesh (2009, p.163) "la transferencia de conocimiento es un proceso de intercambio, tanto del tácito como del explícito, que se produce entre dos agentes, y durante el cual uno de ellos recibe y utiliza el conocimiento proporcionado por el otro". El término "Agente" nos permite estar haciendo referencia a un individuo, a un equipo, a una unidad organizativa, a la organización en sí misma, e incluso a un grupo de organizaciones. El proceso de intercambio implica dos actos complementarios: el de dar o entregar por parte de un agente (la fuente), que se complementa con el de recibir y usar el conocimiento adquirido por el otro (el destinatario). Matsuo (2015, p. 1.189) encontró con sus investigaciones, que la "transferencia es posible a través de los individuos, porque son capaces de crear un contexto de colaboración a partir de la cultura y de la estructura organizacional". En este sentido, Inkpen y Tsang (2005) sugieren que la transferencia de conocimiento se facilita por las interacciones sociales intensas.

\section{Cultura organizacional y transferencia de conocimiento}

Para House et al. (2004) la cultura es un sistema colectivo de motivos, valores, creencias, identidades e interpretaciones, que se 
comparten, de los significados de los acontecimientos evidentes que resultan de las experiencias de los miembros, y se transmiten a través de las generaciones.

Para Fong, Nguyen y Yun (2013), la cultura es la suma de las creencias colectivas y de aquellos otros valores ampliamente compartidos entre los individuos a partir de la transferencia de conocimiento. En opinión de Sáenz, Aramburu y Blanco (2012) la cultura organizacional tiene su base en el conjunto de normas y valores compartidos que controlan y manejan entre sí los miembros de las organizaciones, al tiempo que permiten la participación de los agentes externos. Por su parte, Zhou, Siu y Wang (2010), al mencionar a Deshpande y Wester (1989), definen la cultura corporativa como el patrón de valores compartidos, normas y creencias, que ayudan a la comprensión por parte de sus empleados del funcionamiento y del comportamiento de la organización.

Para Gururajan y Fink (2010), además de los valores y las normas, uno de los factores más importantes en la cultura relacionada con la transferencia de conocimiento es la confianza. Es éste, sin duda, un factor singular, pues puede actuar tanto de barrera como de elemento facilitador. Casson (1997), afirma que la confianza es el resultado de la justificación a priori nacida de la creencia de que alguien honrará sus obligaciones, y no sólo por los incentivos materiales, sino por poseer un alto sentido de lo que significa el compromiso moral. Se supone que este compromiso es racional, porque genera recompensas emocionales. Es esencial que la persona, al recibir el conocimiento transferido, posea valores morales y éticos suficientes como para valorar el generoso gesto del donante. Greengard, $\left(1998^{a}\right)$ opina que la cultura debe crearse a partir de los valores morales y éticos que poseen los empleados, por constituir ellos el activo más importante de una organización.

Para Zhou, Siu y Wang (2010), la confianza basada en la cognición y el afecto son realmente importantes en la transferencia de conocimiento. Del mismo modo, para Abrams et al. (2003), la confianza conduce a aumentar, a menor costo, el intercambio de 
un conocimiento global, al tiempo que aumenta la probabilidad de que aquel que ha sido adquirido por un colega sea entendido de manera suficiente y apropiada como para ser usado.

Tsai y Ghoshal (1998) y Fong, Nguyen y Yun (2013) concuerdan en afirmar que la confianza aumenta la cantidad de información que se puede intercambiar, y se correlaciona positivamente con la transferencia de conocimientos. En consecuencia, la falta de confianza influye negativamente en aquellos casos en los que el conocimiento puede ser efectivamente transferido, hecho que se produce de forma particularmente acusada en situaciones donde hay incertidumbre.

Según Goh (2002), la existencia de una cultura de colaboración, y de una fuerte cooperación, son prerrequisitos necesarios para la transferencia de conocimiento entre los grupos y los individuos. Sin duda, una fuerte cultura de la experimentación con un clima de cooperación y colaboración tendrán una influencia positiva en este tipo de transferencias.

En opinión de Nonaka, Ichijo y Von Krogh (2000), las organizaciones deben construir un contexto propicio y adecuado para la transferencia de conocimiento, donde las personas puedan compartir sus ideas y crear su estilo propio con el fin de que las relaciones sean constructivas y útiles en un lugar de trabajo.

\section{Naturaleza y características del conocimiento tácito y explícito}

La naturaleza y las características de los conocimientos tácitos y explícitos requeridos por una persona para transferir el conocimiento a otra se basan en aquellos mecanismos que lo hacen posible. Choi y Lee (1997), Kogut y Zander (1993), Senker y Faulkner (1996), e Inkpen (2000) y Mazloomi Khamseh y R. Jolly (2008), que entienden como conocimiento tácito aquel cuya característica más significativa es el hecho de que resulta difícil de "articular" por no 
encontrarse codificado debido a su "complejidad" y a su condición de "ambiguo", lo que lo convierte en "dudoso", es decir, en "incierto". La consecuencia directa de estas características de su naturaleza es la dificultad que tiene de ser transferido (Zander y Kogut, 1995; Tsoukas, 1996 y Szulanski, 1996).

Para Zander y Kogut (1995), la condición de tácito se asocia siempre a un conocimiento que resulta difícil poderlo expresar mediante códigos, palabras, números, lenguajes de programación, etc., o dependiente de un sistema y observable en su uso. Y ello debido precisamente a la característica que posee de ser complejo. El conocimiento tácito se puede encontrar en las percepciones subjetivas, las intuiciones, los presentimientos y el "know-how", y frecuentemente sólo puede ser adquirido a través de la experiencia.

La naturaleza tácita del conocimiento puede derivarse de procesos técnicos -destrezas personales y know-how-, o puede emerger de las cogniciones que son más difíciles de expresar y, en consecuencia, de compartir con otros. Tales son los ideales, los valores y los modelos mentales. En esencia, el conocimiento tácito sólo existe debido a las personas y a su habilidad para entender las experiencias de los otros a través del lenguaje común. Es por ello que las organizaciones que poseen una cultura colectiva son más capaces de transferir y absorber el conocimiento tácito. En realidad, puede decirse que la transferencia de conocimiento tácito es típica de culturas colectivistas, como la japonesa, donde las personas generalmente aprenden unas de otras (Canestrino, 2004).

Lubit (2001) plantea diversas soluciones para transmitir el conocimiento tácito sin transformarlo en formas explícitas, por ejemplo, ofreciendo a los trabajadores la oportunidad de observar a los expertos trabajando con problemas. El trabajo en equipo es otra manera de compartir el conocimiento tácito. Un equipo ofrece siempre la oportunidad de observar cómo otros conceptualizan las situaciones, se acercan a los problemas, y generan y evalúan las soluciones. Es indudable que prestando atención, anotando, y analizando las vivencias y las actitudes de respuesta personal es- 
pontánea que surgen derivadas de acontecimientos críticos en el marco de la actividad laboral de una compañía, el buen observador puede captar el conocimiento tácito y, a raíz de ello, desarrollar y programar las rutinas organizacionales.

Von Krogh, Ichijo y Nonaka (2000) proponen algunos mecanismos a través de los cuales se comparte el conocimiento tácito y, en este sentido, la proximidad física y el tiempo son elementos clave, como lo son a su vez las relaciones de interés existentes en el marco en que se mueven los miembros de una comunidad. Entre los mecanismos, encontramos la observación directa, la observación directa acompañada de una descripción verbal, la imitación, y la ejecución conjunta. El común denominador de los diferentes mecanismos está en la observación aguda, en la experimentación, y en el acompañamiento de un mentor que ayuda a que los demás aprendan de sus experiencias.

Por el contrario, el conocimiento explícito es en su naturaleza justo lo opuesto al conocimiento tácito. Este tipo de conocimiento siempre está documentado, es fácil de expresar y está asociado con los hechos y "el saber-qué" (Polanyi, 1967; Garud, 1997 y Zhou, Siu y Wang, 2010). En contraposición al conocimiento tácito, el explícito puede ser definido como aquel que está codificado, por lo que es transferido en un lenguaje formal y sistémico (Polanyi, 1966). Es esta la razón por la que puede encontrarse en los manuales, las bases de datos, los contratos y las licencias, o bien, incorporado a los productos.

La transferencia de conocimiento explícito puede facilitarse a partir de varios mecanismos. Sáenz, Aramburu y Blanco (2012) consideran y valoran a los repositorios de conocimiento como el vehículo apropiado para la integración de los componentes de conocimiento pertenecientes a la base de la organización. Los repositorios actúan como un enlace entre los usuarios y el conocimiento base, operando como un único punto de entrada destinado a ayudar a encontrar la información procedente de las diferentes fuentes de la organización. En esa misma línea facilitadora, los intranets permiten que los empleados tengan acceso a una amplia 
gama de documentos, a las bases de datos, a las noticias y a los enlaces útiles que pueden ayudarles a potenciar su creatividad, o bien, a administrar más eficientemente sus proyectos. Este tipo de vehículos pueden resultar muy efectivos en la generalización del conocimiento, que de ese modo es posible trasplantar fácilmente a otros contextos. Es este un papel que observamos ejercen del mismo modo los blogs y los foros de discusión en línea.

Los avances en las tecnologías de la información han influido significativamente en la capacidad de las personas para compartir y transferir información y conocimiento, y de hacerlo además reduciendo costos y acelerando el proceso de transferencia de mejores prácticas (Elliott y 0' Dell, 1999, citados por Bender y Fish, 2000). Sin duda, los desarrollos en los sistemas de la comunicación, tales como el correo electrónico o las videoconferencias, pueden acelerar la transferencia de la información al reducir los desplazamientos y los viajes. Las videoconferencias, por ejemplo, a menudo son percibidas como un sustituto de las reuniones cara a cara. En algunos casos esto puede ser una solución eficiente; sin embargo, sólo hasta cierto punto.

\section{Estrategias de transferencia de conocimiento}

Joia y Lemos (2010) soportados en Hansen et al. (1999) al proponen dos estrategias para la transferencia de conocimiento organizacional: La codificación y la personalización. En la codificación, el conocimiento es estandarizado, estructurado y almacenado en los sistemas de información. De esta forma, el conocimiento puede ser distribuido con facilidad a todo el personal de la empresa a través de su sistema informático. Podemos decir, por tanto, que la codificación facilita el acceso de la gente a los documentos almacenados en repositorios.

Con esa misma visión Joia y Lemos (2010), afirman que algunos indicadores pertinentes se encuentran reseñados en la literatura científica destinada a la difusión del conocimiento tácito, y que en 
ella se hace hincapié en considerar la componente personal como factor determinante en la transferencia de este tipo de conocimiento.

El conocimiento tácito viene siendo definido como aquel que posee una calidad personal, por lo que es difícil de formalizar y comunicar. Puede decirse que se encuentra encarnado en los individuos. Sería, por ejemplo, el caso de los empleados que poseen unos amplios conocimientos como resultado de los años de experiencia en el trabajo. En las organizaciones, a partir del nombre de la marca establecida, se pueden observar las rutinas compartidas, algo que forma parte de la cultura de la empresa. Visto así, resulta evidente que la transferencia de conocimiento tácito requiere un contexto más rico, es decir, que va más allá de la simple codificación (Daft y Lengel, 1986).

Los aportes anteriores nos han permitido valorar los indicadores de mayor impacto en la transferencia de conocimiento tácito, siendo estos: el lenguaje común, la red de relaciones, la jerarquía, el tipo de entrenamiento, la recompensa, el conocimiento valioso y las comunicaciones. El lenguaje común es un prerrequisito fundamental para que la transferencia de conocimiento se lleve a cabo entre las personas. Por ejemplo: la terminología propia de la actividad de una empresa y la jerga utilizada por los empleados en el ejercicio de su actividad deben ser familiares a todos ellos, puesto que comparten experiencias propias de sus tareas y derivadas de vivencias comunes (Davenport y Prusak, 2003; Disterer, 2003; Haldin-Herrgard, 2000 y Joia y Lemos, 2010).

La red de relaciones va íntimamente unida a la forma de comunicación existente dentro de la empresa, y ello sirve para encontrar el conocimiento deseable y de valor. Esto tiene que ver con la necesidad que siente una persona de encontrar una solución cuando se encuentra frente a un problema que debe resolver. Por lo tanto, este indicador tiene como objetivo el averiguar si es posible la identificación de las personas de la organización que tienen el conocimiento que se necesita para llevar a cabo con eficacia su labor, así como de aquellas otras que necesitan de estos conoci- 
mientos. En este sentido, Davenport y Prusak (2003) afirman que la forma de comunicación dentro de la empresa depende de la red de relaciones internas.

Para Joia (2006), la jerarquía, resulta ser un factor organizativo-burocrático que puede dificultar el proceso de transferencia de conocimiento. Pueden ponerse como ejemplo la existencia de una cadena jerárquica de mando demasiado rígida, la acentuada especialización del trabajo, los procedimientos estandarizados para cada función, o la estructura organizativa compleja e inflexible. Para que la transferencia de conocimiento tácito tenga lugar, la gente debe estar asequible cuando su conocimiento se requiere, independientemente de su posición jerárquica en la organización (Fahey y Prusak, 1998).

El tipo de entrenamiento está relacionado con la contratación de nuevos empleados, el traslado de empleados entre las distintas áreas de la empresa, o la promoción del personal a otros cargos. Es indudable que para ello se requiere un entrenamiento apropiado. Dentro de las estrategias que mejor sirven a la transferencia de conocimiento tácito encontramos a la mentoría y el entrenamiento a través de contactos personales con una alta dedicación de tiempo (Disterer, 2003; Leonard y Sensiper, 1998). En este tipo de formación, a los empleados más experimentados se les anima a transferir sus conocimientos a los empleados más nuevos.

La recompensa como motivación entre los empleados es otro factor a tener en cuenta en la transferencia de conocimiento tácito. Davenport y Prusak (2003) sostienen que, para establecer una cultura constante de intercambio de conocimientos, el uso de incentivos financieros, el aumento del salario, la promoción de los cargos y las propinas, son necesarios. Según Haldin-Herrgard (2000), citado por Joia y Lemos (2010), tanto el conocimiento tácito como el "know-how" deben ser reconocidos en la transferencia de conocimiento a la par que la educación formal.

Para Roberts (2000), el uso de formas valiosas de comunicación adquiere una gran importancia en relación con la transferencia de 
conocimiento tácito. Entre estos medios de comunicación, las conversaciones personales son consideradas las más valiosas, ya que fomentan la retroalimentación mutua e inmediata, utilizando múltiples formas de comunicación. Ahí está, por ejemplo, la demostración de habilidades personales, e incluso el lenguaje corporal (Haldin-Herrgard, 2000; Leonard y Sensiper, 1998).

\section{Metodología}

La información para el estudio se obtuvo de Maloka, Corporación dedicada a fomentar la pasión por el aprendizaje a lo largo de la vida, fortaleciendo los lazos entre ciencia- tecnología-innovación y sociedad, con el fin de enriquecer la cultura ciudadana y aportar al desarrollo sustentable. Está ubicada en Bogotá, Colombia, su fin es aportar a la consolidación de una sociedad y economía basadas en el aprendizaje en el conocimiento y la innovación, que cuente con ciudadanos libres, competitivos, participativos, creativos, innovadores y realizadores, con identidad y autoestima. Al respecto, Posada et al. (2010), afirma que, la apropiación social del conocimiento surge a partir de inquietudes que dan como resultado preguntas destinadas a comprender cómo se apropia la ciencia y la tecnología, de manera que la comunidad haga suyo el conocimiento científico y tecnológico con vistas a mejorar sus condiciones de vida. Las personas participantes fueron 72 altos y medios directivos, incluyendo los empleados que tiene más de 5 años de experiencia ocupando cargos de alta responsabilidad en campos como la investigación, el desarrollo de productos y servicios, el diseño, y el desarrollo de estrategias pedagógicas.

El estudio de los datos se realizó desde el análisis factorial. Esta es una técnica estadística que permite definir grupos de variables que estén altamente correlacionados entre sí, buscando reducir dimensiones. Para saber si realizar un análisis factorial en el instrumento era significativo, se aplicó el test de KMO (Kaiser-MeyerOlkin), pues determina si la técnica de reducción de dimensiones es viable. La fiabilidad del instrumento se midió con el coeficiente 
de alfa de Cronbach, pues permite conocer la precisión de las mediciones que se están tomando en una población determinada en situaciones normales. Al aplicar este coeficiente en el instrumento, se dice que este tiene máxima fiabilidad cuando el alfa es igual a 1 , en caso contrario, si se obtiene un coeficiente 0 , se dice que tiene fiabilidad nula, por ende habrá mayor error en la medición.

La encuesta evalúo (en escala Likert con puntuaciones entre 1 y7) tres grandes partes. La primera relacionada con la cultura organizacional y sus variables como: (a) valores y normas, (b) confianza basada en el saber y el afecto, (c) confianza basada en los valores morales y éticos, (d) creencias colectivas, (e) identidades de acontecimientos, ( $f$ ) cultura de experimentación, (g) cooperación y colaboración, (h) empatía activa, (i) acceso a la ayuda, (j) actitud indulgente. La segunda parte relacionada con la naturaleza y características del conocimiento tácito y explícito requeridas para la transferencia de conocimiento. Esta parte se divide en tres subpartes. La primera sub-parte se relaciona el grado de iniciativas favorecedoras de la transferencia de conocimiento tácito y sus variables como: (a) observación directa a expertos, (b) observación directa y descripción verbal, (c) imitación, (d) experimentación y comparación, (f) práctica común, (g) escribir las "historias que se aprenden" (h) propagando las rutinas. La segunda sub-parte relacionada con la transferencia de conocimiento tácito a partir de varios ejemplos como: (a) percepciones, (b) presentimientos, (c) Know-How, (d) destrezas, (e) juicios de valor a partir de criterios, (f) vivencias y hechos, $(\mathrm{g}$ ) habilidades tácitas producto de las experiencias. La tercera sub-parte relacionada con las herramientas utilizadas como medio para la transferencia de conocimiento explícito y sus variables como: (a) biblioteca técnica on-line, (b) intranets, (c) bases de datos, (d) noticias contenidas en diversos medios, (f) redes, (g) licencias adquiridas (h) contratos, (i) productos y servicios. La tercera parte está relacionada con el grado de aplicación de las estrategias para la transferencia de conocimiento tácito y sus variables como: $(\mathrm{H} 1)$ lenguaje común, $(\mathrm{H} 2)$ red de relaciones conectadas, (H3) directivos en Maloka, (H4) mentoría, (H5) recompensa como motivación y (H6) comunicación, como las conversaciones, la demostración de habilidades personales y el lenguaje corporal. 


\section{Resultados}

En la primera parte, denominada "La cultura organizacional y la transferencia de conocimiento", los encuestados se sometieron a 10 ítems, cuyo objetivo fue evaluar las actitudes, experiencias, creencias y valores que promueven los trabajadores de Maloka en su labor diaria. Para este caso, los 10 ítems obtuvieron medidas de $\mathrm{KMO}$ superiores a 0.8 , por lo que, realizar un análisis factorial en el instrumento resultaba significativo. En general, la medida de KMO fue de 0,89 . Finalmente, se realizó el análisis factorial con dos ejes debido a que retienen el $71 \%$ de la información, obteniendo las siguientes coordenadas, que representamos de forma visible en la siguiente gráfica:

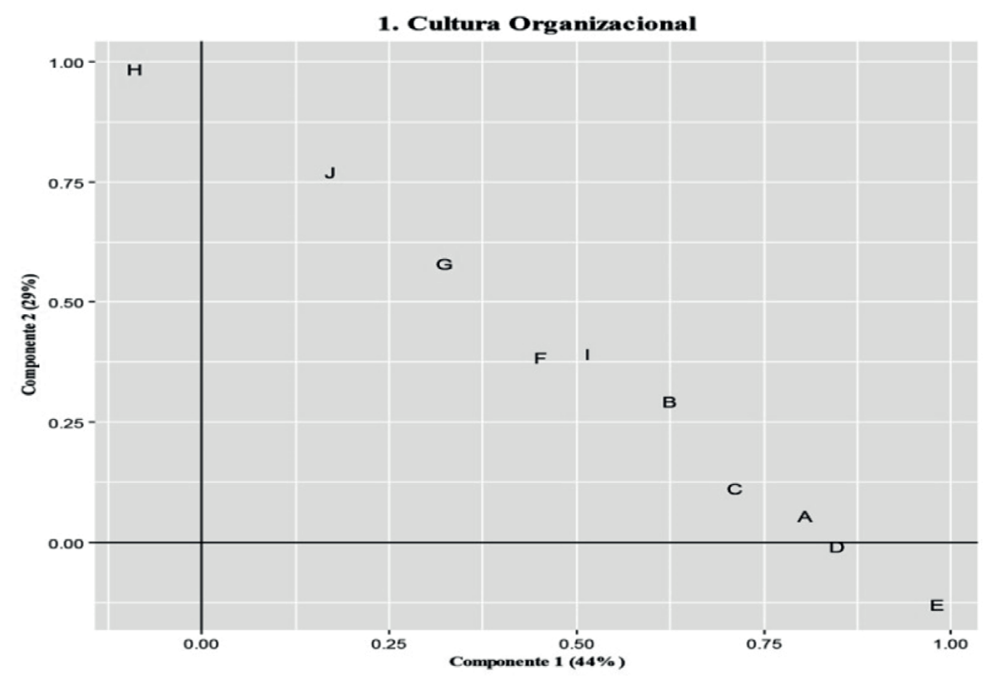

Figura 1. Plano factorial de ítems incluidos para evaluar la cultura organizacional y la transferencia de conocimiento. Fuente. Elaboración propia

El primer eje se puede considerar como el conjunto de valores que cada trabajador debe aportar en el ámbito personal y su entorno laboral dentro de la empresa. En la mencionada figura 1, se observa que, los valores y normas son visibles entre las personas que trabajan en ella, lo que está en completa relación con lo ex- 
presado por Hofstede (1994), Fong, Nguyen y Yun (2013), Sáenz, Aramburu y Blanco (2012), Deshpande y Wester (1989). Por otro lado, existe una alta evidencia de la confianza basada en valores morales y éticos, que concuerda con lo expresado por Gururajan y Fink (2010) y Casson (1997). I gualmente, se observa que, las creencias colectivas y las identidades de acontecimientos que conducen a la transferencia de conocimiento son factores relevantes en la cultura organizacional de la empresa, lo cual está en coherencia con lo planteado sobre el tema por Ralston et al. (1993) y House et al. (2004).

El segundo eje se identifica por la ayuda mutua entre los trabajadores para la transferencia interna de conocimiento. La característica más importante está dada por la empatía activa para comprender a los demás a partir del diálogo. Con estos condicionantes es indispensable generar un estilo de diálogo propio, una conversación auténtica, y de transferir los aprendizajes teniendo en cuenta el nivel y las características que son propias del conocimiento previo de quien ahora lo recibe, que según Nonaka, Ichijo y Von Krogh, (2000) debe ser un factor determinante en la cultura organizacional.

La segunda parte, denominada "Naturaleza y características del conocimiento tácito y explícito requeridas para la transferencia interna de conocimiento", en primer lugar, se mide el grado de aplicación, en la empresa, de la transferencia interna de conocimiento tácito entre los trabajadores. El coeficiente de Cronbach mostró que el instrumento tiene consistencia interna al obtener un alfa de 0,88 , es decir, los ítems presentan una buena fiabilidad. Por otro lado, en el test de $\mathrm{KMO}$ se observó que con un coeficiente de 0,81 obtenido como resultado, era adecuado realizar un análisis factorial. Para este análisis se procedió a realizar el estudio con dos dimensiones; sin embargo, se pudo observar que solo se puede explicar el $54 \%$ de la información con este método, por ello, se decidió efectuar el análisis con tres dimensiones, las cuales retienen el $73 \%$ de la información. Con el desarrollo del análisis en tres dimensiones se pudieron observar las coordenadas que refleja la figura: 


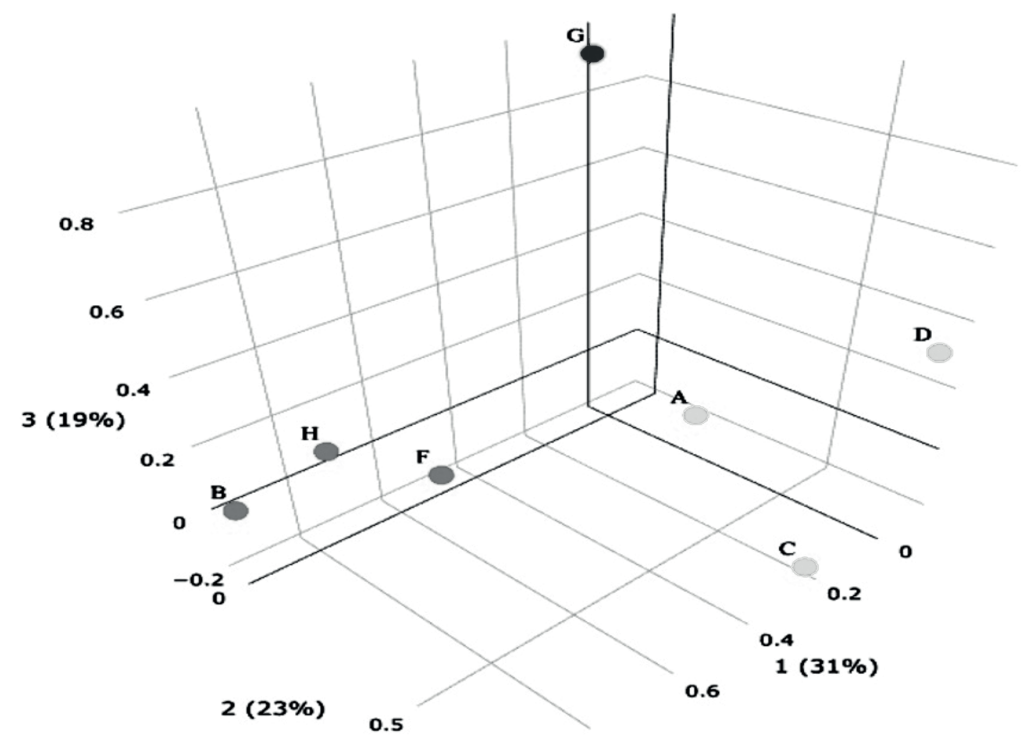

Figura 2. Espacio factorial de ítems incluidos para evaluar el grado de aplicación de iniciativas favorecedoras. Fuente. Elaboración propia

El análisis factorial en tres dimensiones permitió observar cómo se puede agrupar de acuerdo con las calificaciones dadas por los encuestados. Esta clasificación se puede describir de la siguiente forma:

-Los procesos organizacionales y la descripción verbal de los expertos permiten la transferencia interna de conocimiento tácito según el primer eje, en donde se encuentra el $31 \%$ de la información. La característica más importante de este eje viene dada por la transferencia interna de conocimiento tácito a través de la observación directa a los expertos, lo que se relaciona directamente con lo mencionado por Von Krogh, Ichijo y Nonaka (2000).

-En el segundo eje podemos destacar que la transferencia interna de conocimiento tácito se realiza a partir del aprendizaje, que es captado de trabajadores con experiencia a través de la observación directa o la imitación, lo que está acorde con Von Krogh, Ichijo y Nonaka (2000), además del alto impacto que tiene la propagación de las 
rutinas con la transferencia del conocimiento tácito, expresado por Lubit (2001).

-Del tercer eje se puede deducir que los trabajadores realizan un análisis sobre acontecimientos que se presentan en su labor diaria, ejerciendo así un aprendizaje individual para la transferencia interna de conocimiento, siendo ello debido al hecho de escribir y estudiar las "historias que se aprenden". En este sentido, la evidencia está relacionada con los aportes de Lubit (2001). En lo referente a los ítems que evaluaron la transferencia de conocimiento tácito se obtuvo un coeficiente de Cronbach de 0,95, lo cual indica que esos ítems presentan consistencia interna, y alta fiabilidad. La prueba de KMO muestra que todos los ítems se encuentran por encima de 0,90. Debido a ello se considera que el análisis factorial permite un estudio adecuado. En general, se obtuvo un valor de 0,92 para los ejemplos de conocimiento tácito en la transferencia de conocimiento. Esta técnica mostró que el análisis factorial se podía reducir a dos dimensiones, pues capturaba el $76 \%$ de la información con las coordenadas reflejadas en la figura 3.

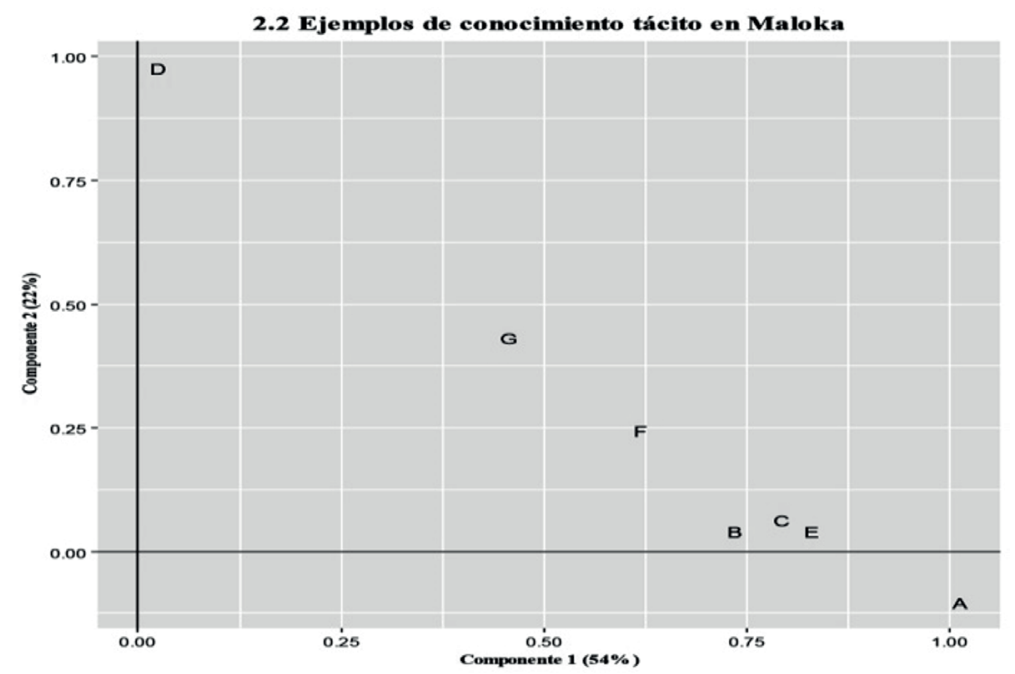

Figura 3. Plano factorial de ítems incluidos para evaluar los ejemplos de transferencia interna de conocimiento tácito. Fuente. Elaboración propia 
Este análisis permite observar que en el primer eje (54\% de la información), se han presentado ejemplos de conocimiento tácito compartidos a partir de las percepciones, los juicios de valor, los presentimientos y el Know-How. En Maloka, se evidencia en alto grado lo mencionado en Zander y Kogut (1995), los cuales aseguran que estos ejemplos de conocimiento tácito son relevantes. Por otro lado, en el segundo eje notamos que compartir y demostrar las destrezas que poseen los trabajadores de Maloka, resulta importante para la transferencia de conocimiento tácito, lo que está en completa concordancia con lo expuesto en Zander y Kogut (1995).

En lo referente a las herramientas que son utilizadas por los trabajadores de la empresa para la transferencia de conocimiento explícito, el alfa de Cronbach fue de 0,82 indicando con esto que el instrumento es fiable. El análisis factorial se realizó con tres ejes, reteniendo el $57 \%$ de la información como se muestra en la figura 4.

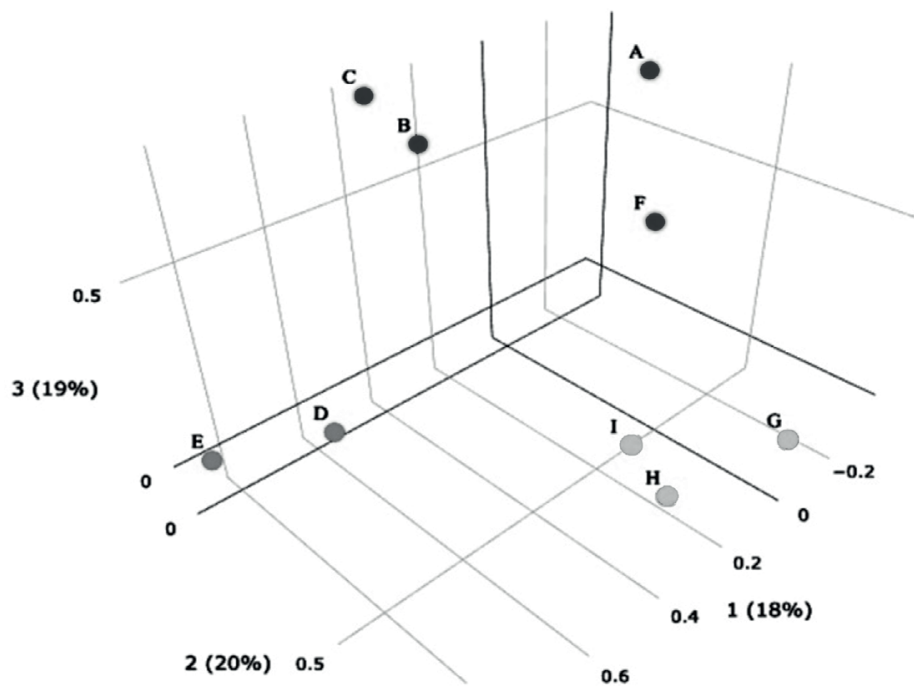

Figura 4. Espacio factorial de ítems incluidos para evaluar las herramientas para la transferencia interna de conocimiento explícito. Fuente. Elaboración propia

-El primer eje se caracteriza por representar las noticias que se publican en distintos medios de comunicación como estrategia para la transferencia interna de conoci- 
miento explícito en la empresa. Este eje retiene el $18 \%$ de la información. Se destaca que los productos y servicios son el insumo más importante. Lo anterior está acorde con Nonaka y Takeuchi (1995) y Blanco (2004).

-El segundo eje, que retiene el $20 \%$ de la información, muestra qué herramientas-los enlaces (redes) y las noticias contenidas en diversos medios-permiten la transferencia de conocimiento explícito entre los trabajadores de la empresa. Lo anterior está acorde con Sáenz, Aramburu y Blanco (2012).

Finalmente, en el tercer eje, que completa el $57 \%$ de la información retenida, es donde se observa que la transferencia de conocimiento explicito entre los trabajadores de Maloka se realiza a través de iniciativas que otorga la navegación por internet, tales como biblioteca online, bases de datos y foros de discusión en línea, etc., lo que concuerda con lo expuesto en Sáenz, Aramburu y Blanco (2012).

Por último, se tiene la tercera parte del instrumento, que consiste en las estrategias o iniciativas para la transferencia interna de conocimiento tácito entre los trabajadores de Maloka. En este caso, el coeficiente de Cronbach mostró una consistencia interna de 0,90 , lo que sin duda significa la buena fiabilidad de la encuesta. De igual forma, se observan valores test de KMO superiores a 0,85, que nos induce a realizar un análisis factorial.

Al realizar la técnica de reducción de dimensiones, se observó que al tener tres ejes en el plano factorial se retiene el $72 \%$ de la información; sin embargo, el tercer eje no ayuda en gran medida debido a su bajo porcentaje de varianza, sólo el $4 \%$, en consecuencia, se desarrolló el análisis con dos dimensiones (68\% de la información), con las coordenadas que se reflejan en la siguiente figura 5 : 


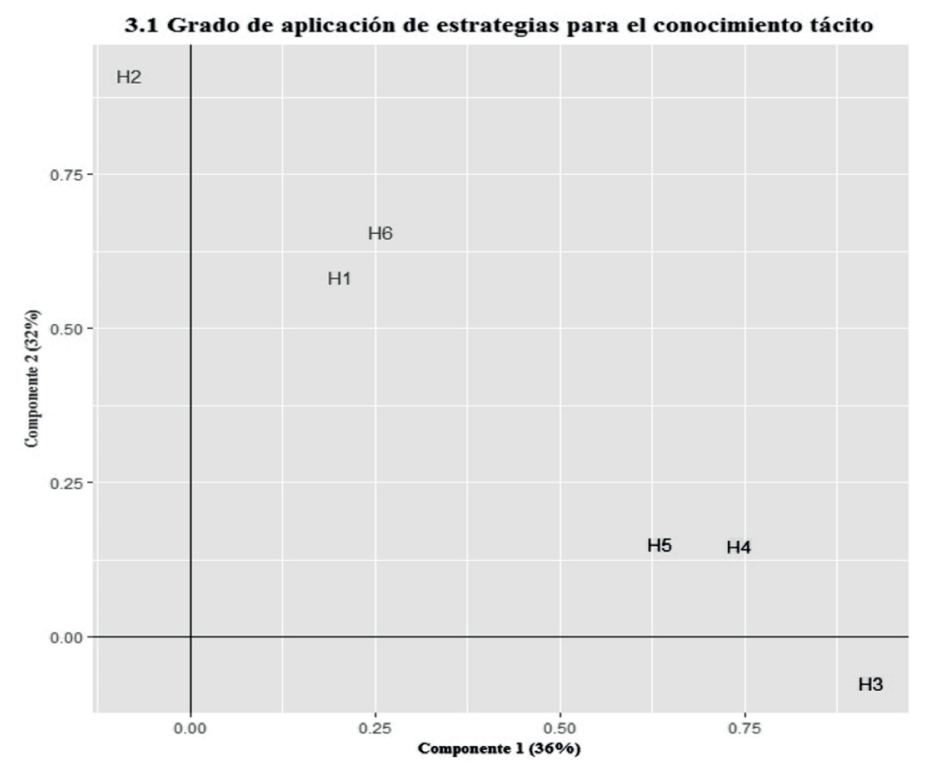

Figura 5. Plano factorial de ítems incluidos para evaluar el grado de aplicación de estrategias para el conocimiento tácito. Fuente. Elaboración propia

El primer eje se puede considerar como el de las estrategias planteadas por los directivos de la empresa y de los expertos con destino a la transferencia interna de conocimiento, ya que hacen posible el acceso al conocimiento tácito que poseen, además de propiciar la mentoría y el entrenamiento destinado a los empleados. De otra parte, la recompensa que se les da a los trabajadores es una oportunidad para motivarse y promover la transferencia de este tipo de conocimiento. Esto es acorde con lo planteado por Fahey y Prusak (1998). Por otro lado, el segundo eje se fundamenta en el hecho de que la estrategia para la transferencia de conocimiento tácito se hace efectiva por medio de redes de relación conectadas, así como por el uso entre las personas de un lenguaje común, ya sea verbal o corporal, a la hora de compartir sus experiencias, lo que propicia el conocimiento de valor. Todo lo anterior se encuentra en consonancia con el discurso en Davenport y Prusak (2003). 


\section{Conclusiones}

En general, los directivos, directivos medios, y empleados con mayor experiencia, muestran que las variables de la cultura organizacional, de la naturaleza del conocimiento tácito y explícito, y de las relacionadas con las estrategias de transferencia son relevantes, y predominan en el comportamiento y la gestión, debido a la naturaleza de Maloka. Sin embargo, en la cultura organizacional se observan vacíos relacionados con la experimentación continua, la cooperación-colaboración, la actitud indulgente en el juicio y el acceso a la ayuda cuando se requiere el conocimiento en una área diferente.

Con respecto a las iniciativas favorecedoras de la transferencia de conocimiento tácito, sigue existiendo el vacío que produce la ausencia de experimentación, aunque se destaca el aprendizaje a través de la observación directa que llevan a cabo los expertos.

\section{Implicaciones prácticas}

Nuestro estudio tiene varias implicaciones importantes de tipo administrativo y carácter práctico. Las organizaciones dedicadas a la divulgación de la ciencia, la tecnología y la innovación deben invertir mucho más en la formación de las personas en materia de gestión de conocimiento, para con ello romper creencias y paradigmas propios de la idiosincrasia personal y social del lugar y del momento. Las creencias y paradigmas arraigados impiden que el conocimiento circule entre las personas, y se siga pensando que su poseedor es quien detenta el poder. La visibilidad de los valores éticos, morales y de confianza en las relaciones conducen a que las estrategias de transferencia de conocimiento sean evidentes en su creación y en los productos y servicios derivados.

Por otra parte, los directivos de las organizaciones, que buscan generar valor en la sociedad a partir de la divulgación de la 
ciencia, la tecnología y la innovación, deben saber que la gestión del conocimiento tácito es crucial para su transferencia interna. El saber identificar en las personas las percepciones subjetivas, las intuiciones, los presentimientos y el "know-how", es un reto para la buena gestión del conocimiento y para saber quién está en posesión de aquel que es significativo.

Finalmente, decir que la adecuada gestión del conocimiento se basa en aplicar las estrategias que hacen posible la transferencia. Sirva como ejemplo la comunicación horizontal, el lenguaje común, la recompensa, el entrenamiento apropiado con ayuda de los expertos y el fomento de una red de relaciones entre las personas. La ayuda de la tecnología como medio es una variable que se debe saber aprovechar, sobre todo, cuando se trata de compartir lo que se tiene en los repositorios de conocimiento, por ejemplo, las historias, los aprendizajes, las lecciones aprendidas y las buenas prácticas.

\section{Limitaciones y futuras Investigaciones}

Esta investigación presenta las siguientes limitaciones: Las medidas se basan en las percepciones de los trabajadores que están en contacto directo con las personas que asisten a Maloka para aprender sobre ciencia, tecnología e innovación. Aquí se puede presentar un sesgo con respecto a los demás participantes, porque tiene internalizadas las variables de las estrategias de transferencia de conocimiento, pero en menos medida aquellas que se relacionan con la transferencia de conocimiento tácito. De otro lado, los directivos tienen apropiadas las variables de la cultura organizacional, pero no saben en qué medida los demás trabajadores las hacen visibles en sus relaciones. Se puede decir que cada persona tiene una percepción subjetiva de las variables confianza, indulgencia en el juicio, ayuda mutua y valores éticos y morales.

Como futuras líneas de investigación, se podría hacer un estudio mediante ecuaciones estructurales, y realizar la indagación de 
las variables culturales a partir de la endoculturación y enculturación en un conjunto de corporaciones colombianas que se dedican a la divulgación de la ciencia, la tecnología y la innovación.

Declaración de conflictos e interés. Los autores no declaran ningún conflicto de interés potencial con respecto a la investigación, la autoría y/o publicación del artículo.

Financiación. Agradecemos a la Universidad Santo Tomás por apoyar en parte esta investigación. También a Maloka por permitirnos hacer las entrevistas personales a la muestra que participó.

\section{Referencias bibliográficas}

Abrams, L., Cross, R., Lesser, E. and Levin, D. (2003). Nurturing interpersonal trust in knowledge-sharing networks. Academy of Management Executive, 17 (4), 64-77.

Barney, J.B. (1991). Firm Resources and Sustained Competitive Advantage. Journal of Management, 17(1). 99 -120.

Beijerse, R. (2000). Knowledge management in small and mediumsized companies. knowledge management for entrepreneurs. Journal of Knowledge Management, 4(2), 162-79.

Bender, S y Fish, A. (2000). The transfer of knowledge and the retention of expertise: the continuing need for global assignments. Journal of Knowledge Management, 4(2), 125-137.

Blanco-Valbuena, C.E. (2004). Impacto de la gestión del conocimiento en las empresas de los Parques tecnológicos Vascos. Universidad de Deusto, San Sebastián, País Vasco. Tesis doctoral.

Blanco- Valbuena, C.E y Peña-Legazkue, I. (2005), Activos Intangibles, acuerdos de colaboración y rendimiento en las empresas de alta tecnología del País Vasco. Cuadernos de Administración, 18 (30), 9-37. Pontificia Universidad Javeriana de Bogotá. 
Blanco-Valbuena, C.E y Bernal, C (2018), Industrias Creativas y Culturales: Estudio desde el Enfoque de la Gestión del Conocimiento. Información Tecnológica, 29 (3), 15-28.

Canestrino, R. (2004). Cross-border knowledge transfer in international strategic alliances: from cultural variations to asymmetric learning process. Proceedings of I-KNOW '04, 30 June-2 July, Graz.

Carlile, P. and Rebentisch, E. (2003). Into the black box: the knowledge transformation cycle. Management Science, 49, 1180-95.

Choi, C.J. and Lee, S.H. (1997). A knowledge-based view of cooperative inter-organizational relationships", in Beamish, P. and Killings, J. (Eds), Cooperative Strategies: European Perspectives, New Lexington Press, San Francisco, CA, 33-58.

Christensen, P.H. (2003). Knowledge Sharing - Time Sensitiveness and Push-pull Strategies in a Non-hyper- organization. EGOS Colloquium, Copenhagen Business School, Copenhagen.

Casson, M. (1997). Information and Organization: A New Perspective on the Theory of the Firm, Clarendon Press, Oxford.

Daft, R.L. y Lengel, R.H. (1986). Organizational information requirements, media richness and structural design. Management Science, 32(5), 55471.

Davenport, T.H. y Prusak, L. (1998). Working Knowledge: How Organizations Manage What They Know. Harvard Business School Press, Boston, MA.

Dawson, R., 2000. Knowledge Capabilities as the Focus of Organizational Development and Strategy. Journal of Knowledge Management, vol. 4, № 4 , pp. 320-327.

Disterer, G. (2003). Fostering knowledge sharing: why and how?", in Reis, A. and Isaias, P. (Eds), Proceedings of the IADIS International Conference e-Society 2003, Lisbon, 219-26.

Fahey, L. and Prusak, L. (1998). The 11 deadliest sins of knowledge management". California Management Review, 40(3), 265-76. 
Fong, Nguyen y Yun Xu. (2013). Knowledge transfer across dissimilar cultures. Journal Knowledge Management, 17 (1), 29-46.

Garvin, D.A. (1993). Building a learning organisation". Harvard Business Review, July-August, 78-91.

Garud, R. (1997). On the distinction between know-how, know-what, and know-why", in Huff, A. and Walsh, J. (Eds), Advances in Strategic Management, JAI Press, Greenwich, CT.

Goh,S.C. (2002). Managing effective knowledge transfer: an integrative framework and some practice implications. Journal Knowledge Management, 6(1), 23-30.

Grant RM. (1996). Toward a Knowledge-Based Theory of the Firm. Strategic Management Journal Winter Special Issue 17,109-122.

Greengard, S. (1998a). Storing, shaping and sharing collective wisdom. Workforce, 77(10), 82-85.

Gururajan y Fink. (2010). Attitudes towards knowledge transfer in an environment to perform. Journal of Knowledge Management, 14 (6), 828-840.

Haldin-Herrgard, T. (2000). Difficulties in diffusion of tacit knowledge in organisations", Journal of Intellectual Capital, 1(4), 357-65.

Hitt MA et al. (2001b). Direct and Moderating Effects of Human Capital on Strategy and Performance in Professional Service Firms: A ResourceBased Perspective. Academy of Management Journal, 43: 449-467.

House, R.J, Hanges, P.J., Javidan, M., Dorfman, P.W. y Gupta, V. (Eds). (2004). Culture, Leadership, and Organizations. The GLOBE Study of 62 Societies, Sage, Thousand Oaks, CA.

Inkpen, A. C. (2000). Learning through joint ventures: A framework of knowledge acquisition. Journal of Management Studies, 37 (7), 10191043.

Inkpen, A.C. y Tsang, E.W.K. (2005). Social capital, networks, and knowledge transfer. Academy of Management Review, 30(1), 146-165. 
Jobson, J. D. (2012). Applied multivariate data analysis: volume II: Categorical and Multivariate Methods. Springer Science \& Business Media.

Kogut, B. and Zander, U. (992). Knowledge of the firm, combinative capabilities and the replication of technology. Organization Science, $3(3), 383-97$.

Kogut, B. and Zander, U. (1993).Knowledge of the firm and the evolutionary theory of the multinational corporation. Journal of International Business Studies, 24(4), 625-46.

Kumar y Ganesh. (2009). Research on knowledge transfer in organizations: a morphology". Journal of knowledge management, 13(4), 161-174.

Leonard, D. and Sensiper, S. (1998).The role of tacit knowledge in group innovation". California Management Review, 40(3), 112-32

Liyanage, C; Elhag, T, Ballal,T; y Li, Q. (2009).Knowledge communication and translation - a knowledge transfer model. Journal of Knowledge Management, 13(3), 118-131.

Lubit, R. (2001). Tacit knowledge and knowledge management: the keys sustainable competitive advantage. Organizational Dynamics, 29(3),164-78.

Marshall, C., Prusak, L. and Shpilberg, D. (1996). Financial risk and the need for superior knowledge management". California Management Review, 38(3), 77-101

Matsuo, M. (2015). Human resource development programs for knowledge transfer and creation: the case of the Toyota Technical Development Corporation". Journal of Knowledge Management, 19(6), 1186-1203.

Mazloomi Khamseh y R. Jolly. (2008). Knowledge transfer in alliances: determinant factors. Journal of Knowledge Management, 12(1), 37-50.

McFadyen, M.A., Semadeni, M. y Cannella, A.A. (2009). Value of strong ties to disconnected others: examining knowledge creation in biomedicine. Organization Science, 20(3), 552-564. 
Nissen, M., Kamel, M. and Sengupta, K. (2000). Integrated analysis and design of knowledge systems and processes. Information Resources Management Journal, 13(1), 24-43.

Nonaka, I. (1994). A dynamic theory of organizational knowledge creation. Organization Science, 5(1), 14-37.

Nonaka, Ichijo y Von Krogh. (2000). Enabling Knowledge Creation. Oxford University Press, Inc.

Penrose, E.T. (1959). The Theory of the Growth of the Firm. John Wiley, New York.

Polanyi, M. (1966).the Tacit Dimension. Routledge and Kegan Paul, London.

Polanyi, M. (1967). The Tacit Dimension, Doubleday, London.

Posada et al. (2010). Deslocalizando la Apropiación Social de la Ciencia y la Tecnología en Colombia: Aportes desde prácticas diversas. Departamento Administrativo de Colciencias, Bogotá.

Roberts, J. (2000). From know-how to show-how? Questioning the role of information and communication technologies in knowledge transfer". Technology Analysis and Strategic Management, 12 (4), 429-43.

Sáenz, Aramburu y Blanco. (2012). Knowledge Sharing and innovation in Spanish and Colombian high-tech firms. Journal Knowledge Management, 6 (6), 919-933.

Seaton, R.A.F. (2002). Knowledge Transfer, Strategic Tools to Support Adaptive, Integrated Water Resource Management Under Changing Conditions at Catchment Scale - A Co-evolutionary Approach, Bedford.

Seashore, S. E., y Yuchtman, E. (1967). Factorial analysis of organizational performance. Administrative Science Quarterly, 377-395.

Senker, J. and Faulkner, W. (1996), "Networks, tacit knowledge and innovation", in Coombs, R., Richards, A., Saviotti, P. and Walsh, V. (Eds. Technological Collaboration, Edward Elgar, Cheltenham, 76-97.

Spender J-C. (1996). Making Knowledge the Basis of a Dynamic Theory of the Firm. Strategic Management Journal Winter Special Issue 17, 45-62. 
Szulanski, G. (1996).Exploring internal stickiness: impediments to the transfer of best practice within the firm", Strategic Management Journal, 17, 27-43.

Teece, D.J., Pisano, G. y Shuen, A.(1997). Dynamic capabilities and strategic management. Strategic Management Journal, 18(7), 509-33.

Thompson, B. (2004). Exploratory and confirmatory factor analysis: Understanding concepts and applications. American Psychological Association.

Tsoukas, H. (1996).The firm as a distributed knowledge system: a constructivist approach". Strategic Management Journal, 17, 11-25.

Tsai, W. and Ghoshal, S. (1998).Social capital and value creation: the role of intrafirm networks". Academy of Management Journal, 41 (4), 464-76.

Van den Hooff, B. y De Ridder, J.A., 2004. Knowledge sharing in context - the influence of organisational commitment, communication climate and $\mathrm{CMC}$ use on knowledge sharing. Journal of Knowledge Management, 8(6), 117-30.

Wernerfelt B.1984. A Resource-Based View of the Firm. Strategic Management Journal 5(2), 171-180.

Zander, U. and Kogut, B. (1995).Knowledge and the speed of transfer and imitation of organizational capabilities: an empirical test". Organization Science, 6, 76-92.

Zahra, S.A. and George, G. (2002), "Absorptive capacity - a review, reconceptualisation and extension". Academy of Management Review, Vol. 27 No. 2, pp. 185-203.

Zhou, Siu y Wang., 2010. Effects of social tie content on knowledge transfer. Journal of Knowledge Management. 14(3), 449-463. 